\title{
No longer 'blob-ology': Cryo-EM is getting into molecular details
}

\author{
ZHU HongTao \& ZHU Ping* \\ National Laboratory of Biomacromolecules, Institute of Biophysics, Chinese Academy of Sciences, Beijing 100101, China
}

Received September 17, 2005; accepted September 26, 2015; published online October 12, 2015

Citation: Zhu HT, Zhu P. No longer 'blob-ology': Cryo-EM is getting into molecular details. Sci China Life Sci, 2015, 58: 1154-1156, doi: 10.1007/s11427-015-4942-0

The structural information of functionally important macromolecular assemblies is a key to molecular biology and is of great interest to structural biologists. Cryo-electron microscopy (cryo-EM), particularly the single particle analysis (SPA), has been regarded as one of the three primary techniques for structure determination, together with X-ray crystallography and nuclear magnetic resonance (NMR). For single particle cryo-EM, a tiny amount $(\sim 3 \mu \mathrm{L})$ of purified biological samples at a concentration around $0.1-2 \mathrm{mg}$ $\mathrm{mL}^{-1}$ are placed onto an EM grid, blotted with a filter paper and rapidly frozen into a thin layer of vitreous ice. The EM grid with frozen samples is then transferred to a transmission electron microscope for imaging. Thousands of micrographs are taken and millions of images of individual macromolecular particles are picked, computationally aligned and averaged to acquire a three-dimensional structure.

Although the techniques and principle of cryo-EM reconstruction had been developed for several decades, the resolution of structures determined by cryo-EM has long been limited to nanometer to sub-nanometer range. The low- to medium-resolution structure, i.e., 3-D density map, generated by cryo-EM reconstruction, although very helpful in understanding the general assembly of the macromolecules, often appears as a "blob" without detailed features. Consequently, cryo-EM technique had been termed as 'blob-ology' for many years.

However, this situation has been changed with a number of breakthroughs made in cryo-EM field in the past two years. In 2013, Liao et al. [1] solved the structure of

*Corresponding author (email: zhup@ibp.ac.cn)
TRPV1, an ion channel membrane protein, at $3.4 \AA$ resolution by single particle analysis. This is thought to be groundbreaking because it opens a door for membrane protein structure determination without crystallization, which is the bottleneck of X-ray crystallography - the currently dominant technique for membrane protein structure determination. At the same time, significant progress had also been made on the cryo-EM structure determination of other macromolecular assemblies or multi-subunit molecular complexes. For example, the structure of the large subunit of a yeast mitochondrial ribosome had been solved by single particle analysis at $3.2 \AA$ resolution [2], which can only be achieved for highly symmetrical icosahedral viruses by cryo-EM previously. At these resolutions, atomic models of macromolecular assemblies or multi-subunit molecular complexes can be built de novo, i.e., solely based on the cryo-EM density maps without the help of atomic structures of the sub-units determined by either X-ray crystallography or NMR technique.

This termed "resolution revolution" in cryo-EM is a consequence of series of advances made recently, both in hardware equipments and data processing procedures. The first, and probably the most important one, is the combination of recently developed direct electron detector device (DDD) with state-of-the-art cryo-electron microscope. Compared with charge-coupled detectors (CCD) which are installed in most of the high-end electron microscopes, the direct electron detector has a noteworthy improvement in the detective quantum efficiency (DQE). The recorded EM image with DDD presents a remarkably better signal to noise ratio (SNR) than that with CCD. This makes the sub- 
sequent image processing much more accurate and efficient. In addition, the newly adopted direct electron recording device makes it possible to correct the beam-induced sample movement. Exposed to charged electron beams in the electron microscope, biological samples buried in vitreous ice are subject to beam-induced movement in stochastic directions, which makes recorded EM micrographs shaky with blurry details and hampers the achievement of atomic structures by subsequent image analysis. By using the newly installed direct electron detectors, dose-fractionated image stacks can be recorded in movie mode and the beam-induced specimen movements can be computationally calculated and corrected, which significantly improve the image quality and greatly help the subsequent structure determination to advance into an atomic resolution level [3,4].

Another critical development attributed to the cryo-EM "resolution revolution" is the improvement of image processing procedures. The intrinsic structural heterogeneity within samples, e.g., the conformational change of biological samples in different states, makes it difficult for researchers to align the images precisely and to resolve the atomic details of macromolecules by cryo-EM. The methods of maximum-likelihood based image alignment and heterogeneous sample classification have greatly improved the resolution of structure determination in single particle analysis. A software package, RELION [5] , which integrates these methods is now widely used in cryo-EM field.

With the help of these dramatic technical advances, numerous cryo-EM structures at atomic resolution level have been achieved for a range of specimens, from small membrane proteins to multi-subunits macromolecular complexes. For small membrane proteins, the structure of human $\gamma$-secretase, an asymmetric membrane protein with molecular weight of $170 \mathrm{kD}$, has been recently determined at $3.4 \AA$ resolution [6]. For multi-subunits macromolecular complexes, a series of long-awaiting structures have been solved at near-atomic resolution, for example, the structure of dynactin complex, a 23-subunit complex, has been determined at the $4.0 \AA$ resolution [7] and the structure of a yeast spliceosome, a 37 subunits complex, has been resolved at $3.6 \AA$ resolution [8]. Having been controversial for several decades, the structure of $30 \mathrm{~nm}$ chromatin fiber, an essential component of nucleus, was also solved by cryo-EM at $11 \AA$ resolution with $\mathrm{CCD}$ images [9]. Without the recent advances in cryo-EM technique, to determine the structures of these huge, sometimes flexible, macromolecular complexes would be highly challenging, if not impossible.

Currently, the resolution record of structure determination by single particle cryo-EM has been set to $2.2 \AA$ [10], which is now beginning to rival X-ray crystallography. No longer a "blob-ology", cryo-EM is on the way to play one of the major roles in structure determination of macromolecular complexes at atomic resolution.

Nevertheless, various efforts are still needed to make cryo-EM a powerful, convenient and attractive tool for structure determination.

(i) Improve structure determining resolution to a higher level. New generations of electron detectors with thinner chip and faster readouts are being developed, which may yield a better DQE close to the physical limit. More efficient motion correction algorithms, new types of EM grid or supporting films are also being developed to further reduce or even completely stop the beam-induced sample movement. A better resolution, e.g., $1.5 \AA$, will make cryo-EM useful for structure-based drug or vaccine design, and attractive to the pharmaceutical industry.

(ii) Work with more flexible and structurally heterogeneous samples. The intrinsic flexibility or dynamic characteristics of biological samples post a great challenge to the structure determination at atomic resolution. Better image classification methods are being developed to deal with biological samples with larger amounts of structural heterogeneity. It would be indispensible if cryo-EM could reveal the structure dynamics of macromolecular complexes through extensive classifications of hundreds of millions of particles in different status.

(iii) Break the bottleneck of biochemical sample preparation. While high-throughput cryo-EM structure determination is on the way, the biochemical sample preparation becomes the main bottleneck. An efficient method to produce sufficient samples for cryo-EM structure determination is clearly in eager need. With relatively less sample required for cryo-EM, extracting endogenous macromolecular assemblies from cells or tissues and solving their structures in native status would become feasible.

(iv) Make cryo-EM less expensive and more accessible. Currently, the establishment and maintenance of a state-of-the-art cryo-EM facility is highly costly and the whole process of cryo-EM structure determination is quite experience demanding. The whole field would benefit greatly from improved accessibility of the cryo-EM equipment and technique.

With numerous technology advances on the road, cryo-EM is now revolutionizing the structural biology and paving the way for a "golden era".

1 Liao M, Cao E, Julius D, Cheng Y. Structure of the TRPV1 ion channel determined by electron cryo-microscopy. Nature, 2013, 504: 107-112

2 Amunts A, Brown A, Bai XC, Llacer JL, Hussain T, Emsley P, Long F, Murshudov G, Scheres SH, Ramakrishnan V. Structure of the yeast mitochondrial large ribosomal subunit. Science, 2014, 343: 1485-1489

3 Li X, Mooney P, Zheng S, Booth CR, Braunfeld MB, Gubbens S, Agard DA, Cheng Y. Electron counting and beam-induced motion correction enable near-atomic-resolution single-particle cryo-EM. Nat Methods, 2013, 10: 584-590

4 Bai XC, Fernandez IS, McMullan G, Scheres SH. Ribosome structures to near-atomic resolution from thirty thousand cryo-EM particles. eLife, 2013, 2: e00461

5 Scheres SH. RELION: implementation of a Bayesian approach to 
cryo-EM structure determination. J Struct Biol, 2012, 180: 519-530

6 Bai XC, Yan C, Yang G, Lu P, Ma D, Sun L, Zhou R, Scheres SH, Shi Y. An atomic structure of human gamma-secretase. Nature, 2015, 525: 212-217

7 Urnavicius L, Zhang K, Diamant AG, Motz C, Schlager MA, Yu M, Patel NA, Robinson CV, Carter AP. The structure of the dynactin complex and its interaction with dynein. Science, 2015, 347: 1441-1446

8 Yan C, Hang J, Wan R, Huang M, Wong CC, Shi Y. Structure of a yeast spliceosome at 3.6-angstrom resolution. Science, 2015, 349: 1182-1191

9 Song F, Chen P, Sun D, Wang M, Dong L, Liang D, Xu RM, Zhu P, Li G. Cryo-EM study of the chromatin fiber reveals a double helix twisted by tetranucleosomal units. Science, 2014, 344: 376-380

10 Bartesaghi A, Merk A, Banerjee S, Matthies D, Wu X, Milne JL, Subramaniam S. $2.2 \AA$ resolution cryo-EM structure of beta-galactosidase in complex with a cell-permeant inhibitor. Science, 2015, 348: 1147-1151

Open Access This article is distributed under the terms of the Creative Commons Attribution License which permits any use, distribution, and reproduction in any medium, provided the original author(s) and source are credited. 